COMMENT. Abnormalities in the basal ganglia associated with perinatal asphyxia were described in clinical and neuropathological studies in the mid- $20^{\text {th }}$ century and earlier, especially in relation to cerebral palsy. (Norman RM. In: Greenfield's Neuropathology. Baltimore, Williams and Wilkins, 1963; pp. 390-397; Christensen E, Melchior J. Cerebral palsy - A clinical and neuropathological study. Clin Dev Med 1967;25:1; Ingram TTS. Paediatric Aspects of Cerebral Palsy. Edinburgh, E \& S Livingstone, 1964). Status marmoratus (etat marbre), marbling of the basal ganglia is a well-recognized pathological finding in children with athetoid CP. Regarded initially as a prenatal developmental anomaly (Vogt C, Vogt O. J Psychol Neurol 1919;24:1, cited in Ingram 1964) etat marbre is now considered a sequel to perinatal birth anoxia, and associated with a history of asphyxia or trauma or, sometimes, kernicterus or status epilepticus. The thalami and other brain regions may also be affected. Characteristically, shrinkage of the basal ganglia is accompanied by coarse networks of myelinated nerve fibers, termed dysmyelination or hypermyelination. Stained by Weigert's method, the myelin marbled appearance is revealed as alternating light and dark areas in the putamen and caudate nuclei. Some well known pediatric neurologists have contributed to our understanding of the syndrome of status matmoratus and $\mathrm{CP}$, including Crothers B. Amer J Dis Child 1921;22:145; and Ford FR. Diseases of the Nervous System in Infancy, Childhood and Adolescence. $4^{\text {th }}$ ed. Springfield, IL. Charles C Thomas, 1960.

In the Hammersmith study report, status marmoratus is not mentioned as a possible pathology involving the basal ganglia of patients who developed athetoid cerebral palsy or in the 8 infants who died. Presumably, autopsies were not obtained. MRI descriptions of the basal ganglia abnormalities in the neonatal period showed swelling and homogeneous appearance, not shrunken and marbled. Status marmoratus may develop as a late finding in older CP patients. My colleague, Dr Mark Wainwright provided the following references to the MRI in patients with cerebral palsy: Mizuguchi M and Takashima S (Neuropathology 2002;22:85-89) report that radiological techniques are unable to visualize or identify pathological changes of status marmoratus; and reporting results of the European CP Study, Bax M and associates (JAMA 2006;296:1602-1608) found basal ganglia abnormalities in $12.8 \%$, described as reduction in volume and increased signal in a child aged 18 months with dyskinetic CP. MRI obtained at 18 months of age or later was normal in $11 \%$ of children with $\mathrm{CP}$.

\title{
PRE-TERM AND PERINATAL PREDICTORS OF NEONATAL HIPPOCAMPAL VOLUMES
}

Hippocampal volumes of 184 preterm (PT) and 32 full-term (FT) infants were measured by segmental MRI at term equivalent age in an investigation of correlations of preterm hippocampal volume, perinatal risk factors, and neurodevelopmental outcome, at University of Melbourne, Victoria, and other centers in Australia; St Louis and Boston, USA; and Geneva, Switzerland. No significant differences between PT and FT infant hippocampal volumes were detected, after controlling for head size. Factors associated with significantly smaller hippocampal volumes included white matter injury, exposure to postnatal steroids, and treatment with indomethacin. Smaller PT hippocampal volumes correlated with impaired cognitive and psychomotor development measured by the Bayley Scales at 2 years of age, 
after correcting for head size and sex. (Thompson DK, Wood SJ, Doyle LW, et al. Neonate hippocampal volumes: prematurity, perinatal predictors, and 2-year outcome. Ann Neurol May 2008;63:642-651). (Resapond: Deanne K Thompson, BSc(Hons), Howard Florey Institute, Level 2, Alan Gilbert Building, 161 Barry Street, Carlton South, VIC 3053, Australia).

COMMENT. The hippocampus, part of the limbic lobe, originally linked mainly with olfactory function, is now considered important in memory, spatial function, and cognition. Hippocampal volume reduction reported in children with chromosome 22q11.2 deletion syndrome is correlated with severity of cognitive impairment. (Deboer T et al. Behav Brain Funct 2007;3:54). Severe memory impairment is reported in a 5-year-old child with marked hippocampal atrophy after prolonged status epilepticus. (Jambaque I et al. Dev Med Child Neurol 2007;49:398-399). The authors of the above report in infants recommend further MRI studies in older children to determine the role of the hippocampus in the high rate of cognitive impairment in preterm infants tested at a later age. They advocate interventions to decrease white matter damage and overuse of postnatal steroids and indomethacin in preterm infants, factors linked to smaller hippocampal volume.

\section{VERY PRETERM BIRTH, CEREBELLAR DEVELOPMENT AND NEUROPSYCHOLOGICAL OUTCOME IN ADOLESCENCE}

Cerebellar volumes were measured on structural MRI at adolescence and adulthood in 65 preterm individuals (born before 33 weeks' gestation), and a term-born comparison group, in a study at King's College, Great Ormond Street Hospital, and University College, London; and Seoul National University College of Medicine, Korea. Cerebellar volumes in late adolescence and adulthood (mean age $18.6 ; \mathrm{SD}=1.02$ ) were $3.11 \%$ and significantly smaller than measurements during early adolescence (mean age 15 years; $\mathrm{SD}=1.43$ ) in the preterm group $(\mathrm{P}=0.000)$, whereas cerebellar volumes increased $0.44 \%$, but did not change significantly with age in the control group $(\mathrm{P}=0.612)$. The changes in cerebellar volume correlated with tests of behavior and cognitive function. High General Health Questionnaire (GHQ)-12 scores, a self-reporting wellbeing test (eg feeling worthless, poor concentration), indicative of increased risk of mental health problems, correlated with reduction in cerebellar volume during late adolescence and young adulthood. Cerebellar volume correlated positively with full scale, verbal and performance IQ in early adolescence in the very preterm group but not the term-born group. Correlations with IQ were not maintained after controlling for white matter volume. (Parker J, Mitchell A, Kalpakidou A, et al. Cerebellar growth and behavioural \& neuropsychological outcome in preterm adolescents. Brain May 2008;131:1344-1351). (Respond: Matthew Allin, Kung's College London, Division of Psychological Medicine and Psychiatry, Institute of Psychiatry, London, UK. E-mail: matthew.allin@iop.kcl.ac.uk)

COMMENT. A decrease in cerebellar volume occurring between mean age 15 years and 18.6 years in very preterm individuals is correlated with impaired feelings of wellbeing, but a correlation with IQ deficits is not significant when controlled for white matter volume. These findings corroborate previous reports of cerebellar involvement in cognitive and neurobehavioral disorders. (Murakami JW et al. Arch Neurol 1989;46:689-694). 